$\left(\mathrm{Cl}\right.$ and $\mathrm{ClO}$ ) is removed from the $\mathrm{ClO}_{x}$ cycle. Surprisingly, the two reactions of the $\mathrm{ClO}_{x}$ cycle, that is $\mathrm{Cl}+\mathrm{O}_{3} \rightarrow \mathrm{ClO}$ $+\mathrm{O}_{2}$ and $\mathrm{O}+\mathrm{ClO} \rightarrow \mathrm{Cl}+\mathrm{O}_{2}$, do not introduce large errors, because their rates have been carefully measured in the laboratory. The first measurements of $\mathrm{Cl}+\mathrm{O}_{3}$ and $\mathrm{O}+\mathrm{ClO}$ rates by Bemand, Clyne and Watson at Queen Mary College in 1973 (see J. Chem. Soc., Faraday I, 69, 1356; 1973; Clyne and Watson, J. Chem. Soc. Faraday I, $70,2250 ; 1974)$ have been only slightly altered as a result of subsequent work in the USA and in this laboratory.

Significant uncertainty $( \pm$ a factor of 1.7) in the prediction of ozone depletion is also introduced by the onedimensional model used by the NAS panel to approximate the distribution and transport of the chemical species involved. This model is equivalent to averaging the concentrations, motions and chemical reactions over latitude and longitude, leaving only their dependences upon altitude and time. This approximation is considered to be a good one. For instance, the equivalents of averaged gas transport rates appear in the model as the vertical cddy mixing or transport coefficient, which depends only on the altitude.

One further very important question concerns the effects of an increased ultraviolet intensity (UV-B, 280 to $320 \mathrm{~nm}$ ) at ground level on animals and plants. The biological effects of UV-B are not as extensively dealt with by the NAS reports as are other aspects. This may be due to the relative lack of knowledge in this area. It is clearly to be identified as a priority area for future work on the CFM-ozone problem. However, in general terms, the deleterious effects of UV-B radiation on living cells are well known. For example, there is a clear causal connection between the more common (but less serious) forms of skin cancer (basal and squamous cell carcinomas) and UV-B exposure. The connection between the less common, but often fatal malignant melanoma and UV-B, is suggestive but not certain, according to the NAS report.

Release of pollutants other than CFM, which can also affect ozone levels, is another topic requiring extensive future work. Among halogen compounds, further work on methyl chloride formed by the burning of vegetation, is indicated. Also, Crutzen has suggested that methyl chloroform $\left(\mathrm{CH}_{3} \mathrm{CCl}_{3}\right)$, whose manufacture as a degreasing agent is considerable and possibly increasing, may eventually cause ozone depletion comparable to that of C.FM 11 and 12. Bromine compounds, released for example as methyl bromide in soil fumigants, are probably not produced in large enough quantities to have major effects. However, $\mathrm{Br}$ atoms and $\mathrm{BrO}$ enter into a similar, but more effective cycle to that of $\mathrm{Cl}$ and $\mathrm{ClO}$.

$$
\mathrm{Br}+\mathrm{O}_{:} \rightarrow \mathrm{BrO}+\mathrm{O}_{:,}, \mathrm{O}+\mathrm{BrO} \rightarrow \mathrm{Br}+\mathrm{O}_{:} .
$$

The rate constants for the bromine reactions are not particularly well known. Nitrous oxide, apparently re-
THE in vitro cultivation of malaria parasites has been possible for many years but only one or a few successive cycles of development have been achieved and these successes have been mainly with the malaria parasites of monkeys. The amount of physiological and biochemical knowledge that has accumulated from such studies has been vast and it cannot be disputed that much of it is directly or indirectly applicable to chemotherapeutic and immunological studies aimed at the control of malaria. What is desperately required, however, is the long term continuous cultivation of human malaria parasites which cannot be maintained in standard laboratory animals. For some time now there have been rumours of the successful cultivation of Plasmodium falciparum in the United States and now two independent reports have appeared, giving the longawaited experimental details (Trager and Jensen, Science, 193, 673; 1976; and Haynes et al., page 767 in this issue of Nature). There can now be no doubt that the continuous cultivation of $P$. falciparum can be achieved in simple media and that it should shortly be possible to obtain the yields of parasites necessary for the production of experimental vaccines.

Both media are remarkably simple. Trager and Jensen use a modification of an earlier medium (RPMI 1640 with HEPES buffer and serum) for the cultivation of $P$. coatneyi from monkeys (Moore et al., J. Am. med. Ass., 199, 519; 1967) but substitute human serum for the monkey serum originally used. In essence, the medium

\section{Cultivation of human malaria}

from F. E. G. Cox

flows over a shallow bed of red blood cells which are disturbed as little as possible in an atmosphere of low $\mathrm{O}_{2}$ and high CO.. The technical details are still being modified and Trager and Jensen began by using $7 \% \mathrm{CO}_{2}$ and $5 \% \mathrm{O}_{2}$ but changed this to $1 \%, \mathrm{O}_{2}$ in later experiments with better results. The technique of Haynes et al. is more traditional, involving a supplemented medium 199 with $1 \%$ foetal bovine serum in an atmosphere of $3 \% \mathrm{CO}$. No doubt many other ways will be found to grow $P$. falciparum, and Trager and Jensen report success using their medium in a candle jar (a container in which a candle has burned and extinguished).

The most important features of the cultivation techniques described seem to be the high levels of $\mathrm{CO}$. and the fact that the number of red cells infected rarely rises above $1 \%$, thus preventing the culture medium from becoming too acidic as a result of the metabolic activities of the parasites, which is a problem inherent in this kind of $\mathrm{cx}$ periment. Although the multiplication of parasites recorded is low (a fourfold increase per cycle is assumed by Trager and Jensen) the numbers of parasites available for collection can be considerable if parallel cultures are set up and initiated from diluted cultures at the end of each cyclc. Haynes et al. obtained eleven successive cultures and Trager and Jensen more than twentyfive (and Trager has since reported continuous cultivation over $105 \mathrm{~d}$, implying fifty or more cycles) so there is no reason why such cultures should not be maintained indefinitely.

The technique described by Trager and Jensen looks more promising than that of Haynes et al., but the latter authors have made a major advance by showing that their cultures can be initiated from frozen blood samples. It should therefore be possible to initiate a culture and to freeze stabilates from that culture for the initiation of others. This would save the lives of countless owl monkeys (Aotus trivirgatus), which at the moment are the only sources of laboratory maintained $P$. falciparum, and also standardise experiments carried out in different laboratories.

The implications of these experiments are considerable, for as well as the biochemical and physiological observations which are now made feasible it should be possible to grow the numbers of merozoites (the stages that pass from one cell to another) needed for producing vaccines. The only homologous vaccine with real potential, in monkeys at least, is that based on merozoites (Mitchell, Butcher and Cohen, Immunology, 29, 397; 1975) and the production of a suitable vaccine free from viruses is only possible from cultured parasites. With 96 million cases of malaria each year and with $P$. falciparum being the major killer the production and evaluation of such a vaccine should not be delayed. 